Pis'ma v ZhETF

\title{
Pseudoscalar mesons and their radial excitations from the Effective Chiral Lagrangian
}

\author{
S. M. Fedorov ${ }^{1)}$, Yu. A. Simonov ${ }^{2}$ \\ Institute of Theoretical and Experimental Physics, 117218, Moscow, B.Cheremushkinskaya 25, Russia
}

Submitted June 23, 2003

\begin{abstract}
Effective Chiral Lagrangian is derived from QCD in the framework of Field Correlator Method. It contains the effects of both confinement and chiral symmetry breaking due to a special structure of the resulting quark mass operator. It is shown that this Lagrangian describes light pseudoscalar mesons, and Gell-Mann-OakesRenner relations for pions, eta and K mesons are reproduced. Spectrum of radial excitations of pions and K mesons is found and compared to experimentally known masses.
\end{abstract}

'PACS: 11.15.Tk,11.30.Rd,12.38.-t,12.39.Fe,14.40.Aq

1. QCD is known to possess two highly nontrivial fea'tures at low temperatures, namely confinement and chi'ral symmetry breaking (CSB). At some critical tempera'ture phase transitions of deconfinement and chiral sym'metry restoration occur. From lattice calculations it is known that these two phase transitions take place at 'the same temperature [1, 2]. The fact that two critical temperatures coincide was not fully understood so far. 'This work is a continuation of a series of papers [3, 4, 5], 'where it is argued that CSB occurs due to confinement in a very nontrivial way.

It was shown in 3 that effective four-quark interaction leading to spontaneous chiral symmetry breaking, 'occurs in QCD due to confinement, and is associated 'with the QCD string. Thus, CSB is closely connected to confinement. In this approach the Effective Chiral Lagrangian (ECL) containing fields of light pseudo-scalar mesons is derived from QCD Lagrangian. This is done by integrating out gluon fields and performing bosonisation. At the same time confinement is taken into account through specific form of gluon-field correlators.

As a result, expanding in powers of (derivatives) of bosonic fields, one obtains the ECL similar to the celebrated Gasser-Leutwyler Lagrangian [6], however in the nonlocal form 3 .

We expand ECL in powers of meson fields, and reproduce standard Gell-Mann-Oakes-Renner relations, while meson masses are zero in the chiral limit. It is shown that the vanishing of meson masses happens due to cancellation of two terms in Green's functions of mesons. Poles of Green's function corresponding to radial excitations of pseudoscalar mesons are displaced from the masses, obtained in Hamiltonian approach

\footnotetext{
2) fedorov@heron.itep.ru

2)simonov@heron.itep.ru
}

without CSB effects (see e.g. 7] and references therein), and are shifted down by less than $15 \%$.

2. We consider Euclidean partition function for quarks and gluons in the presence of external classical currents $v_{\mu}, a_{\mu}, s$ and $p$

$$
\begin{aligned}
Z & =\int D A D \bar{\psi} D \psi \exp \left[-\left(S_{0}+S_{1}+S_{\mathrm{int}}+S_{\text {g.f. }}+S_{\mathrm{gh}}\right)\right], \\
S_{0} & =\frac{1}{4} \int d^{4} x\left(F_{\mu \nu}^{a}\right)^{2}, \\
S_{1} & =-i \int d^{4} x \bar{\psi}^{f}\left(\hat{\partial}+\hat{v}+\gamma_{5} \hat{a}+s+i \gamma_{5} p\right)^{f g} \psi^{g}, \\
S_{\mathrm{int}} & =-\int d^{4} x \bar{\psi}^{f} g \hat{A}^{a} t^{a} \psi^{f} .
\end{aligned}
$$

Here $f, g=1,2,3$ are flavor indices, $t^{a}$ are generators of color SU(3) group, $\operatorname{tr} t^{a} t^{b}=\delta^{a b} / 2, a=1, \ldots 8$. $S_{\text {g.f. }}$. and $S_{\text {gh }}$ are gauge fixing and ghost terms.

Next, we use the generalized contour gauge 8,9

$$
A_{\mu}(x)=\int_{0}^{1} d s \frac{\partial z_{\nu}(s, x)}{\partial s} \frac{\partial z_{\rho}(s, x)}{\partial x_{\mu}} F_{\nu \rho}(z(s))
$$

Here $z_{\nu}(s, x)$ belongs to a set of contours, with properties: $z_{\nu}(0, x)=x_{0}, z_{\nu}(1, x)=x_{\nu}, x_{0}$ is a fixed point. In what follows the exact position of contours is unimportant for our analytical results, while for numerical estimates we will assume that contours are chosen to minimize the spectrum (and area of the string world sheet), to be called the minimal set of contours.

The reason we use contour gauge is that it allows to express gauge field $A_{\mu}$ through field strength tensor 
$F_{\mu \nu}$. Now we are in position to integrate out gluon field $A_{\mu}$, expressing the result in terms of field correlators:

$$
\begin{aligned}
& Z=\iint D \bar{\psi} D \psi \exp \left[-\left(S_{1}+S_{\text {eff }}\right)\right] \\
& \exp \left[-S_{\text {eff }}\right]=\left\langle\exp \left[-S_{\text {int }}\right]\right\rangle_{A}
\end{aligned}
$$

We use cluster expansion to evaluate this average over gluon fields

$$
\begin{aligned}
& \left\langle\exp \left[-S_{\mathrm{int}}\right]\right\rangle_{A}=\exp \left(\sum_{n} \frac{(-1)^{n}\left\langle\left\langle S_{\mathrm{int}}^{n}\right\rangle\right\rangle}{n !}\right), \\
& \left\langle\left\langle S_{\mathrm{int}}\right\rangle\right\rangle=\left\langle S_{\mathrm{int}}\right\rangle_{A} \equiv 0, \\
& \left\langle\left\langle S_{\mathrm{int}}^{2}\right\rangle=\left\langle S_{\mathrm{int}}^{2}\right\rangle_{A}-\left\langle S_{\mathrm{int}}\right\rangle_{A}^{2}=\left\langle S_{\mathrm{int}}^{2}\right\rangle_{A},\right. \\
& \ldots
\end{aligned}
$$

It is clear that gauge invariant quantities like spectrum and Green's functions computed with the help of $S_{\text {eff }}$ do not depend on the chosen contours, if all terms of cluster expansion are retained in (4). In what follows we will use the Gaussian approximation, and consider only first two terms in cluster expansion, $n=1,2$. As was shown in [10, 11] the Gaussian approximation on minimal surfaces is accurate within few percent. Thus, we have

$$
\begin{aligned}
& S_{\text {eff }}=-\frac{1}{2} \int d^{4} x d^{4} y \bar{\psi}_{i \alpha}^{f}(x) \psi_{j \beta}^{f}(x) \bar{\psi}_{k \gamma}^{g}(y) \psi_{l \delta}^{g}(y) \times \\
& \times \int_{0}^{1} d s d t \frac{\partial z_{\rho}(s, x)}{\partial s} \frac{\partial z_{\lambda}(s, x)}{\partial x_{\mu}} \frac{\partial z_{\rho^{\prime}}(t, y)}{\partial t} \frac{\partial z_{\lambda^{\prime}}(t, y)}{\partial y_{\nu}} \times \\
& \times\left\langle\left[F_{\rho \lambda}(z(s, x))\right]_{i j}\left[F_{\rho^{\prime} \lambda^{\prime}}(z(t, y))\right]_{k l}\right\rangle_{A}\left(\gamma^{\mu}\right)_{\alpha \beta}\left(\gamma^{\nu}\right)_{\gamma \delta} .
\end{aligned}
$$

Here $i, j, k, l$ are color indices, $\alpha, \beta, \gamma, \delta$ are spinor indices. Inserting parallel transporters $\Phi\left(x, x_{0}\right)$ and $\Phi\left(y, x_{0}\right)$, which are identically equal to unity in contour gauge, one finally gets expression for effective action

$$
\begin{aligned}
& S_{\text {eff }}=-\frac{1}{2} \int d^{4} x d^{4} y \bar{\psi}_{i \alpha}^{f}(x) \psi_{j \beta}^{f}(x) \bar{\psi}_{k \gamma}^{g}(y) \psi_{l \delta}^{g}(y) \times \\
& \quad \times\left(\delta_{j k} \delta_{i l}-\frac{1}{N_{c}} \delta_{i j} \delta_{k l}\right) J_{\alpha \beta \gamma \delta}(x, y), \\
& J_{\alpha \beta \gamma \delta}(x, y)=\left(\gamma_{\mu}\right)_{\alpha \beta}\left(\gamma_{\nu}\right)_{\gamma \delta} J_{\mu \nu}(x, y), \\
& J_{\mu \nu}(x, y)=\frac{1}{N_{c}^{2}-1} \times \\
& \quad \times \int_{0}^{1} d s d t \frac{\partial z_{\rho}(s, x)}{\partial s} \frac{\partial z_{\lambda}(s, x)}{\partial x_{\mu}} \frac{\partial z_{\rho^{\prime}}(t, y)}{\partial t} \frac{\partial z_{\lambda^{\prime}}(t, y)}{\partial y_{\nu}} \times \\
& \quad \times \operatorname{tr}\left\langle F_{\rho \lambda}\left(z(s, x), x_{0}\right) F_{\rho^{\prime} \lambda^{\prime}}\left(z(t, y), x_{0}\right)\right\rangle_{A}, \\
& F\left(u, x_{0}\right) \equiv \Phi\left(x_{0}, u\right) F(u) \Phi\left(u, x_{0}\right) .
\end{aligned}
$$

Performing bosonization, and keeping only scalarisoscalar and pseudoscalar-isovector (corresponding to pions, $K$ and $\eta$ mesons) terms, one arrives at the quarkmeson Lagrangian (see [4] for details):

$$
\begin{aligned}
& Z=\int D \bar{\psi} D \psi D M_{s} D \phi_{a} \exp \left[-S_{\mathrm{QM}}\right], \\
& S_{\mathrm{QM}}=-\int d^{4} x d^{4} y\left[\bar{\psi}_{i \alpha}^{f}(x) \times\right. \\
& \times\left(i\left(\hat{\partial}+\hat{v}+\gamma_{5} \hat{a}+s+i \gamma_{5} p\right)_{\alpha \beta}^{f g} \delta^{(4)}(x-y)+\right. \\
& \left.+i M_{s}(x, y) \hat{U}_{\alpha \beta}^{f g}(x, y)\right) \psi_{i \beta}^{g}(y)- \\
& \left.-2 N_{f}(J(x, y))^{-1} M_{s}^{2}(x, y)\right], \\
& J(x, y)=J_{\mu \mu}(x, y), \\
& \hat{U}_{\alpha \beta}^{f g}(x, y)=\exp \left(i \gamma_{5} t_{a} \phi_{a}(x, y)\right)_{\alpha \beta}^{f g} .
\end{aligned}
$$

It is now straightforward to integrate out quark fields to obtain the Effective Chiral Lagrangian:

$$
\begin{aligned}
& Z=\int D M_{s} D \phi_{a} \exp \left[-S_{\mathrm{ECL}}\right], \\
& S_{\mathrm{ECL}}=2 N_{f} \int d^{4} x d^{4} y(J(x, y))^{-1} M_{s}^{2}(x, y)+W(\phi), \\
& W(\phi)=N_{c} \operatorname{tr} \ln \left[i\left(\hat{\partial}+\hat{v}+\gamma_{5} \hat{a}+s+i \gamma_{5} p\right)+\right. \\
& \left.\quad+i M_{s}(x, y) e^{i \gamma_{5} t_{a} \phi_{a}(x, y)}\right] .
\end{aligned}
$$

Here tr refers to flavor and spinor indices and to space coordinates. $M_{s}$ is the effective quark mass operator, and $\phi_{a}$ are fields of pseudoscalar mesons (up to the dimensional factor $2 / f, f$ is the decay constant, $\left.\phi_{a}=2 \pi_{a} / f\right)$.

Classical equations of motion are given by

$$
\begin{aligned}
& N_{c} \operatorname{Tr}\left(-S_{\phi}(x, y) M_{s}(x, y) e^{i \gamma_{5} t_{a} \phi_{a}(x, y)} \gamma_{5} t_{a}\right)=0 \\
& N_{c} \operatorname{Tr}\left(i S_{\phi}(x, y) e^{i \gamma_{5} t_{a} \phi_{a}(x, y)}\right)- \\
& \quad-4 N_{f}(J(x, y))^{-1} M_{s}(x, y)=0 \\
& S_{\phi}(x, y) \equiv\left\langle x\left|\frac{1}{i \hat{\partial}+i M_{s} e^{i \gamma_{5} t_{a} \phi_{a}}}\right| y\right\rangle
\end{aligned}
$$

This leads to solutions

$$
\begin{aligned}
& \phi_{a}^{(0)}(x, y)=0, \\
& M_{s}^{(0)}(x, y)=\frac{N_{c}}{4 N_{f}} J(x, y) \operatorname{Tr}(S(x, y)), \\
& \left.S(x, y) \equiv S_{\phi}(x, y)\right|_{\phi=0} .
\end{aligned}
$$

Second equation in (10) is a nonlinear equation for $M_{s}^{(0)}$, and the existence of a nontrivial solution is a manifestation of the chiral symmetry breaking, since $M_{s}^{(0)}$ is scalar. The system of equations (10) for $M_{s}^{(0)}$ and $S(x, y)$ was considered in 12 for the special case of 
heavy-light mesons, and it was shown that it has a confining scalar solution for $M_{s}^{(0)}(x, y) \simeq \sigma\left|\vec{x}-\vec{x}_{0}\right| \delta^{(3)}(x-y)$ for large distance $\left|\vec{x}-\vec{x}_{0}\right|$ from quark to (heavy) antiquark at the point $\vec{x}_{0}$. It is clear that the same type of solution occurs at large interquark distances for lightlight mesons, which means that confinement and CSB occur spontaneously and simultaneously from the nontrivial solution of the system (10).

3. We consider ECL (8), expanding it in powers of the field $\phi_{a}$ up to the second order, and we introduce current quark masses $\mathcal{M}_{f} \equiv \operatorname{diag}\left(m_{u}, m_{d}, m_{s}\right)$. Neglecting external currents, one obtains

$$
\begin{aligned}
& W(\phi)=N_{c} \operatorname{tr} \ln \left[i\left(\hat{\partial}+\mathcal{M}_{f}+M_{s} e^{i \gamma_{5} t_{a} \phi_{a}}\right)\right]= \\
& =N_{c} \operatorname{tr} \ln \left[i\left(\hat{\partial}+\mathcal{M}_{f}+M_{s}\right)+\right. \\
& \left.+\left(-M_{s} \gamma_{5} t_{a} \phi_{a}-\frac{i}{2} M_{s} t_{a} t_{b} \phi_{a} \phi_{b}\right)\right], \\
& W^{(2)}(\phi)=-\frac{N_{c}}{2} \operatorname{tr}\left[i S \cdot\left(M_{s} t_{a} t_{b} \phi_{a} \phi_{b}\right)+\right. \\
& \left.+S \cdot\left(M_{s} t_{a} \phi_{a}\right) \cdot \gamma_{5} S \gamma_{5} \cdot\left(M_{s} t_{b} \phi_{b}\right)\right] .
\end{aligned}
$$

Taking trace in flavor indices, (see 4 for details), one arrives at the following expression for the term quadratic in meson fields:

$$
\begin{aligned}
& W^{(2)}(\phi)=-\int d^{4} x d^{4} y\left[W_{\pi \pi}(x, y) \phi_{\pi}^{*}(x) \phi_{\pi}(y)+\right. \\
& +W_{K K}(x, y) \phi_{K}^{*}(x) \phi_{K}(y)+ \\
& +W_{K^{0} K^{0}}(x, y) \phi_{K^{0}}^{*}(x) \phi_{K^{0}}(y)+ \\
& \left.+\frac{1}{2} \sum_{i, j=3,8} W_{i j}(x, y) \phi_{i}(x) \phi_{j}(y)\right]
\end{aligned}
$$

where, for example,

$$
\begin{aligned}
& W_{\pi \pi}(x, y)=\frac{N_{c}}{4} \operatorname{Tr}\left[S_{u}(x, y) M_{s}(y) \gamma_{5} S_{d}(y, x) \gamma_{5} M_{s}(x)+\right. \\
& \left.\quad+i S_{u}(x, x) M_{s}(x) \delta^{4}(x-y)+(u \leftrightarrow d)\right]
\end{aligned}
$$

$S_{u}, S_{d}$ and $S_{s}$ are quark propagators (10) with current mass of corresponding quark in the denominator. Here we have taken the local limit of nonlocal operators $M_{s}(x, y) \rightarrow M_{s}(x) \delta^{4}(x-y), \phi(x, y) \rightarrow \phi(x)$, which is obtained when gluonic correlation length $T_{g}$ in the correlator $\langle F F\rangle$ tends to zero.

Two terms in (13) correspond to connected and disconnected diagrams, which cancel each other in the zero momentum limit. This cancellation is exact in the chi- ral limit. To be more precise, quadratic term for zero momentum (i.e. when $\phi(x)=$ const) takes the form:

$$
\begin{aligned}
& \left.W^{(2)}(\phi)\right|_{\text {zero momentum }}= \\
& =\frac{N_{c}}{4} \int d^{4} x\left[\frac{m_{u}+m_{d}}{2} \operatorname{Tr}\left(-i S_{u}(x, x)-i S_{d}(x, x)\right) \phi_{\pi}^{*} \phi_{\pi}+\right. \\
& \quad+\ldots]+O\left(m^{2}\right) .
\end{aligned}
$$

Taking into account that

$\langle\bar{\psi} \psi\rangle_{M}=-i\langle\bar{\psi} \psi\rangle_{E}=-\frac{1}{Z} \frac{\delta Z[v, a, s, p]}{\delta s(x)}=N_{c} \operatorname{Tr}(i S(x, x))$,

where $\langle\bar{\psi} \psi\rangle_{M}$ and $\langle\bar{\psi} \psi\rangle_{E}$ denote quark condensate in Minkovski and Euclidean space respectively, and that $\phi_{a}=2 \pi_{a} / f, f$ is the decay constant, $\pi_{a}$ are physical meson fields, one finds

$$
\begin{aligned}
& f^{2} M_{\pi^{ \pm}}^{2}=2 \hat{m}|\langle\bar{q} q\rangle|+O\left(m^{2}\right) \\
& f^{2} M_{\pi^{0}}^{2}=2 \hat{m}|\langle\bar{q} q\rangle|-\varepsilon+O\left(\varepsilon^{2}\right)+O\left(m^{2}\right) \\
& f^{2} M_{K^{ \pm}}^{2}=\left(m_{u}+m_{s}\right)|\langle\bar{q} q\rangle|+O\left(m^{2}\right) \\
& f^{2} M_{K^{0}}^{2}=\left(m_{d}+m_{s}\right)|\langle\bar{q} q\rangle|+O\left(m^{2}\right) \\
& f^{2} M_{\eta_{8}}^{2}=\frac{2}{3}\left(\hat{m}+2 m_{s}\right)|\langle\bar{q} q\rangle|+\varepsilon+O\left(\varepsilon^{2}\right)+O\left(m^{2}\right) .
\end{aligned}
$$

Here $\hat{m}=\left(m_{u}+m_{d}\right) / 2$. We have neglected differences between quark condensates for different flavors, corrections are of order of $m_{q}^{2}$. Small mixing of $\phi_{3}$ and $\phi_{8}$ states due to isospin symmetry breaking (proportional to $m_{u}-m_{d}$ ) yields a correction $\varepsilon$ to pion and $\eta$ meson masses:

$$
\begin{aligned}
& \pi^{0} \sim \cos (\delta) \phi_{3}+\sin (\delta) \phi_{8}, \\
& \eta_{8} \sim-\sin (\delta) \phi_{3}+\cos (\delta) \phi_{8}, \\
& \tan (2 \delta)=\sqrt{3} \frac{m_{d}-m_{u}}{2 m_{s}-\left(m_{u}+m_{d}\right)} \\
& \varepsilon=\frac{|\langle\bar{q} q\rangle|\left(m_{u}-m_{d}\right)^{2}}{4 m_{s}-2\left(m_{u}+m_{d}\right)}, \\
& \delta \simeq 0.6^{\circ} .
\end{aligned}
$$

Thus, ECL (8) leads to correct Gell-Mann-OakesRenner relations for all light pseudoscalar mesons.

4. Let us now consider Green's functions of mesons, generated by the pseudoscalar currents:

$$
\begin{aligned}
& \mathfrak{G}_{a b}(x, y)=\left\langle J_{a}^{5}(x) J_{b}^{5}(y)\right\rangle=\frac{1}{Z} \frac{\delta^{2} Z}{\delta p_{a}(x) \delta p_{b}(y)}, \\
& J_{a}^{5}(x)=\bar{\psi}(x) \gamma_{5} t_{a} \psi(x), \\
& p^{f g}(x)=p_{a}(x) t_{a}^{f g} .
\end{aligned}
$$


From the ECL one obtains

$$
\begin{aligned}
& \mathfrak{G}_{a b}(x, y)=\frac{1}{Z} \int D M_{s} D \phi_{a} \exp \left[-S_{\mathrm{ECL}}\right] \times \\
& \times\left[N_{c} \operatorname{Tr}\left(S_{\phi}(x, y) \gamma_{5} t_{a} S_{\phi}(y, x) \gamma_{5} t_{b}\right)-\right. \\
& \left.\quad-N_{c}^{2} \operatorname{Tr}\left(S_{\phi}(x, x) \gamma_{5} t_{a}\right) \operatorname{Tr}\left(S_{\phi}(y, y) \gamma_{5} t_{b}\right)\right]
\end{aligned}
$$

Taking $M_{s}$ at the stationary point (10) and expanding $S_{\phi}$ in terms of $\phi$ around $\phi_{a}^{(0)}=0$, one finds:

$S_{\phi}(x, y)=S(x, y)+\int d^{4} z S(x, z) \cdot M_{s}(z) \gamma_{5} \phi_{a}(z) t_{a} \cdot S(z, y)$

As argued in 4], the coupling constant $g_{\pi q \bar{q}}$ is of the order of $N_{c}^{-1 / 2}$, and thus in large $N_{c}$ limit pion exchanges are suppressed. This allows to neglect pion fields in connected terms (first term in equation (19)), and consider only one pion exchange in the disconnected term. Resulting expression will contain two terms, both of order of $N_{c}$.

Taking into account, that $S(x, y)=$ $\operatorname{diag}\left(S_{u}(x, y), S_{d}(x, y), S_{s}(x, y)\right)$ is diagonal in flavor, one finds:

$$
\begin{aligned}
& \mathfrak{G}_{\pi^{+} \pi^{+}}(x, y)=\frac{N_{c}}{2} \operatorname{Tr}\left(S_{d}(x, y) \gamma_{5} S_{u}(y, x) \gamma_{5}\right)- \\
& -\frac{N_{c}^{2}}{4} \int d^{4} z_{1} d^{4} z_{2} \operatorname{Tr}\left(S_{u}\left(x, z_{1}\right) M_{s}\left(z_{1}\right) \gamma_{5} S_{d}\left(z_{1}, x\right) \gamma_{5}\right) \times \\
& \quad \times \operatorname{Tr}\left(S_{d}\left(y, z_{2}\right) M_{s}\left(z_{2}\right) \gamma_{5} S_{u}\left(z_{2}, y\right) \gamma_{5}\right) G_{\pi \pi}^{\phi}\left(z_{1}, z_{2}\right) .
\end{aligned}
$$

Other Green functions differ only in flavor indices. Here $G_{\pi \pi}^{\phi}\left(z_{1}, z_{2}\right)=\left\langle\phi_{\pi}^{*}\left(z_{1}\right) \phi_{\pi}\left(z_{2}\right)\right\rangle$ is the propagator of pion field. This formula can be illustrated with the Feynman diagram:

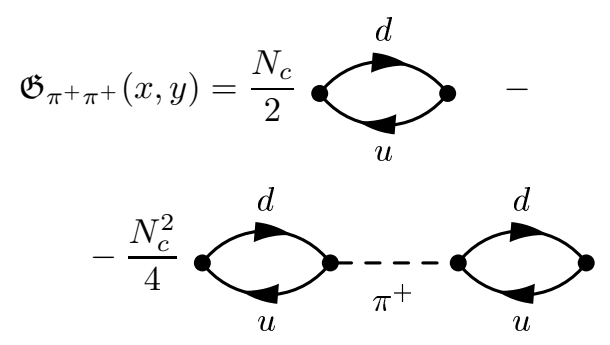

To find the pion propagator one should consider equations (1213):

$$
\begin{aligned}
& \left(G_{\pi \pi}^{\phi}(x, y)\right)^{-1}=W_{\pi \pi}(x, y)= \\
& =\frac{N_{c}}{4} \operatorname{Tr}\left[2 S_{d}(x, y) M_{s}(y) \gamma_{5} S_{u}(y, x) M_{s}(x) \gamma_{5}+\right. \\
& \left.+i\left(S_{d}(x, x) M_{s}(x)+S_{u}(y, y) M_{s}(y)\right) \delta^{4}(x-y)\right]
\end{aligned}
$$

Going over to the momentum space $\mathfrak{G}(x, y)=$ $\int d^{4} k /(2 \pi)^{4} \exp (i k(x-y)) \mathfrak{G}(k)$, where we have also taken into account that Green function is translational invariant, i.e. depends only on $(x-y)$, one has:

$$
\begin{aligned}
& \mathfrak{G}_{\pi^{+} \pi^{+}}(k)=\frac{N_{c}}{2} G_{\pi^{+} \pi^{+}}^{(0)}(k)- \\
& \quad-\frac{N_{c}^{2}}{4} G_{\pi^{+} \pi^{+}}^{(0, \mathrm{M})}(k) G_{\pi^{+} \pi^{+}}^{\phi}(k) G_{\pi^{+} \pi^{+}}^{(0, \mathrm{M})}(k), \\
& G_{\pi^{+} \pi^{+}}^{(0)}(x, y) \equiv \operatorname{Tr}\left(S_{d}(x, y) \gamma_{5} S_{u}(y, x) \gamma_{5}\right), \\
& G_{\pi^{+} \pi^{+}}^{(0, \mathrm{M})}(x, y) \equiv \operatorname{Tr}\left(S_{u}(x, y) M_{s}(y) \gamma_{5} S_{d}(y, x) \gamma_{5}\right) .
\end{aligned}
$$

Due to Eqs. (14-16) the pion propagator (23) has pole at $k^{2}=-M_{\pi^{ \pm}}^{2}$ and can be rewritten as

$$
\begin{aligned}
& G_{\pi^{+} \pi^{+}}^{\phi}(k)=\frac{2}{N_{c}} \frac{1}{G_{\pi^{+} \pi^{+}}^{(0, \mathrm{MM})}(k)-G_{\pi^{+} \pi^{+}}^{(0, \mathrm{MM})}\left(k^{2}=-M_{\pi^{ \pm}}^{2}\right)}, \\
& G_{\pi^{+} \pi^{+}}^{(0, \mathrm{MM})}(x, y) \equiv \operatorname{Tr}\left(S_{u}(x, y) M_{s}(y) \gamma_{5} S_{d}(y, x) M_{s}(x) \gamma_{5}\right)
\end{aligned}
$$

As argued in 4, all three Green functions $G_{\pi^{+} \pi^{+}}^{(0)}$, $G_{\pi^{+} \pi^{+}}^{(0, \mathrm{M})}$, and $G_{\pi^{+} \pi^{+}}^{(0, \mathrm{MM})}$ have the same set of poles, which are poles of the quark model (i.e. confined $\bar{q} q$ system without chiral symmetry breaking) in pseudo-scalar channel, and can be represented as

$$
\begin{aligned}
& G_{\pi^{+} \pi^{+}}^{(0)}(k)=-\sum_{n=0}^{\infty} \frac{c_{n}^{2}}{k^{2}+m_{n}^{2}}, \\
& G_{\pi^{+} \pi^{+}}^{(\mathrm{M}, \mathrm{M})}(k)=-\sum_{n=0}^{\infty} \frac{c_{n} c_{n}^{(\mathrm{M})}}{k^{2}+m_{n}^{2}}, \\
& G_{\pi^{+} \pi^{+}}^{(0, \mathrm{MM})}(k)=-\sum_{n=0}^{\infty} \frac{\left(c_{n}^{(\mathrm{M})}\right)^{2}}{k^{2}+m_{n}^{2}},
\end{aligned}
$$

where

$$
\begin{aligned}
& c_{n}=\sqrt{\frac{m_{n}}{2}} \varphi_{n}(0), \\
& c_{n}^{(\mathrm{M})}=\sqrt{\frac{m_{n}}{2}} M(0) \varphi_{n}(0),
\end{aligned}
$$

$\varphi_{n}(\mathbf{r})$ is the $3 \mathrm{D}$ spin-singlet wave function of $\bar{q} q$ system, and $M(0)$ is a constant related to mass operator $M_{s}$, evaluated in [5] through $\sigma=0.18 \mathrm{GeV}^{2}$ and $T_{g}=1 \mathrm{GeV}^{-1}$ to be $M(0)=148 \mathrm{MeV}$. Thus one has for the pion Green function:

$$
\begin{aligned}
& \mathfrak{G}_{\pi^{+} \pi^{+}}(k)=-\frac{N_{c}}{2} \frac{\Psi(k)}{\left(k^{2}+M_{\pi^{ \pm}}^{2}\right) \Phi(k)}, \\
& \Psi(k)=\sum_{n, m=0}^{\infty} \frac{c_{n}^{2}\left(c_{m}^{(\mathrm{M})}\right)^{2}}{\left(k^{2}+m_{n}^{2}\right)\left(m_{m}^{2}-M_{\pi}^{2}\right)}, \\
& \Phi(k)=\sum_{n=0}^{\infty} \frac{\left(c_{n}^{(\mathrm{M})}\right)^{2}}{\left(k^{2}+m_{n}^{2}\right)\left(m_{n}^{2}-M_{\pi}^{2}\right)} .
\end{aligned}
$$


Clearly, the Green function (28) has pole at $k^{2}=$ $-M_{\pi^{ \pm}}^{2}$, and all poles of quark model are cancelled, since the same set of poles appears in functions $\Psi(k)$ and $\Phi(k)$. The radial excitations of $\pi^{ \pm}$meson are given by zeros of the function $\Phi(k)$. In the first approximation it reads:

$$
\begin{aligned}
k^{2} & =-m_{1}^{2}\left(1+\delta_{1}\right), \\
\delta_{1} & =-\frac{1}{m_{1}^{2}} \frac{c_{1}^{2}\left(m_{1}^{2}-m_{0}^{2}\right)\left(m_{0}^{2}-M_{\pi^{ \pm}}^{2}\right)}{c_{1}^{2}\left(m_{0}^{2}-M_{\pi^{ \pm}}^{2}\right)+c_{0}^{2}\left(m_{1}^{2}-M_{\pi^{ \pm}}^{2}\right)}
\end{aligned}
$$

Masses of $K^{0}, \bar{K}^{0}$ radial excitations can be found from (29) with the exchange of $\pi$ meson mass and reference spectrum with those for $K$ mesons. Numerical results for masses of radial excited states are presented in the next section.

It should be mentioned, that $\eta$ meson requires separate consideration, because of it's mixing with isoscalar state $\eta^{\prime}$, which is different for mesons and their radial excitations. Study of $\eta$ meson spectrum and mixings is planned for the future work.

5. Masses and wave functions of reference spectrum can be obtained from the QCD string Hamiltonian (first derived in 13, 14, 15, and improved to take into account quark self-energy in [16]), where we have put $L=0$ :

$$
H=\frac{m_{1}^{2}}{2 \mu_{1}}+\frac{m_{2}^{2}}{2 \mu_{2}}+\frac{\mu_{1}+\mu_{2}}{2}+\frac{p_{r}^{2}}{2 \tilde{\mu}}+\sigma r-\frac{4}{3} \frac{\alpha_{s}}{r} .
$$

Here $m_{1}$ and $m_{2}$ are current masses of quarks, $\mu_{1}$ and $\mu_{2}$ are einbein parameters, to be found from the eigenvalues of Hamiltonian (30) via $\partial \bar{M}_{n}\left(\mu_{1}, \mu_{2}\right) / \partial \mu_{1}=0$, $\partial \bar{M}_{n}\left(\mu_{1}, \mu_{2}\right) / \partial \mu_{2}=0 ; \tilde{\mu}=\mu_{1} \mu_{2} /\left(\mu_{1}+\mu_{2}\right)$, and $p_{r}$ is the radial component of momentum. This Hamiltonian allows to find spin averaged masses and wave functions. Spin-spin interaction can than be taken into account as a perturbation:

$$
\begin{gathered}
M_{n}=\bar{M}_{n}\left(\mu_{1}, \mu_{2}\right)+\frac{32 \pi \alpha_{s_{1}} \overrightarrow{s_{1}} \overrightarrow{s_{2}}}{9 \mu_{1} \mu_{2}}\left|\varphi_{n}(0)\right|^{2}+ \\
\quad+\frac{4}{3}\left\langle\frac{\alpha_{s}}{r^{3}}\right\rangle \frac{\left\langle 3\left(\overrightarrow{s_{1}} \vec{n}\right)\left(\overrightarrow{s_{2}} \vec{n}\right)-\overrightarrow{s_{1}} \overrightarrow{s_{2}}\right\rangle}{\mu_{1} \mu_{2}}+\Delta_{\mathrm{SE}} \\
\Delta_{\mathrm{SE}}=-\frac{2 \sigma}{\pi}\left(\frac{1}{\mu_{1}}+\frac{1}{\mu_{2}}\right) \eta ; \quad \eta \sim 0.9 \div 1 .
\end{gathered}
$$

$\Delta_{\mathrm{SE}}$ is the quark self-energy term due to field correlators. Factor $\eta$ is a calculable function of current quark masses, but is close to 1 when quark masses are small.
Next, we plug in numbers:

$$
\begin{aligned}
m_{u} & =0.005 \mathrm{GeV}, m_{d}=0.009 \mathrm{GeV}, m_{s}=0.17 \mathrm{GeV}, \\
\sigma & =0.18 \mathrm{GeV}^{2}, \\
\alpha_{s} & =0.3
\end{aligned}
$$

and taking into account that lowest state is shifted exactly to physical value of meson mass (due to GellMann-Oakes-Renner relations), we finally get the following chiral shift of reference (quark model) spectra:

pions:

$$
\begin{array}{ll}
\pi(1 S) & 0.51 \mathrm{GeV} \rightarrow 0.14 \mathrm{GeV}(\text { exact }) \\
\pi(2 S) & 1.51 \mathrm{GeV} \rightarrow 1.25 \mathrm{GeV}(\exp : 1.3 \mathrm{GeV}) \\
\pi(3 S) & 2.18 \mathrm{GeV} \rightarrow 1.98 \mathrm{GeV}(\exp : 1.8 \mathrm{GeV})
\end{array}
$$

K mesons:

$$
\begin{aligned}
& K(1 S) \quad 0.63 \mathrm{GeV} \rightarrow 0.49 \mathrm{GeV} \text { (exact) } \\
& K(2 S) \quad 1.57 \mathrm{GeV} \rightarrow 1.43 \mathrm{GeV}(\exp : 1.46 \mathrm{GeV}) \\
& K(3 S) \quad 2.21 \mathrm{GeV} \rightarrow 2.1 \mathrm{GeV}(\exp : 1.83 \mathrm{GeV})
\end{aligned}
$$

It can be seen, that masses of radial excitations are shifted by less than $15 \%$, and the shifts are small for high excitations. Moreover, one can estimate that $\delta M / M(4 S) \simeq 0.05$ for pions and $\delta M / M(4 S) \simeq 0.04$ for $\mathrm{K}$ mesons. Also, one can see that masses of higher excitations and the slope of radial Regge trajectory differ from the experimental. The reason is that Hamiltonian (30) does not take into account effects of string breaking, which are important for highly excited states, since they have large spatial extent. As was shown in [17] the inclusion of string breaking effects does not violate the linearity of radial trajectories, which is in agreement with experimental data. ${ }^{3)}$

6. Effective Chiral Lagrangian (8) is derived directly from QCD Lagrangian in the framework of Vacuum Correlators Method. This Lagrangian correctly describes light pseudoscalar mesons, which are massless in the chiral limit and satisfy Gell-Mann-Oakes-Renner relations when quark masses are nonzero.

Poles of quark model Green's function are shifted by less then $15 \%$, and the shift is small for highly excited states.

The authors are grateful to A.M. Badalian for valuable comments and discussions.

\footnotetext{
3) The notion of linear radial Regge trajectories is connected to the Veneziano-type "linear dual models" [18.
} 
This work is supported by NSh-1774.2003.2 grant and INTAS-00110 and INTAS-00366 grants.

1. F. Karsch, arXiv:hep-lat/9903031

2. J. M. Carmona, M. D'Elia, L. Del Debbio, A. Di Giacomo, B. Lucini and G. Paffuti, Nucl. Phys. Proc. Suppl. 106, 607 (2002) arXiv:hep-lat/0110058.

3. Yu. A. Simonov, Phys. Rev. D 65, 094018 (2002) arXiv:hep-ph/0201170.

4. Yu. A. Simonov, arXiv:hep-ph/0302090

5. Yu. A. Simonov, arXiv:hep-ph/0305281

6. J. Gasser and H. Leutwyler, Annals Phys. 158, 142 (1984);

J. Gasser and H. Leutwyler, Nucl. Phys. B 250, 465 (1985);

for a review see H. Leutwyler, arXiv:hep-ph/9406283

7. Yu. A. Simonov, arXiv:hep-ph/9911237

8. S. V. Ivanov and G. P. Korchemsky, Phys. Lett. B 154, 197 (1985);

S. V. Ivanov, G. P. Korchemsky and A. V. Radyushkin, Yad. Fiz. 44, 230 (1986).

9. V. I. Shevchenko and Yu. A. Simonov, Phys. Lett. B 437, 146 (1998) arXiv:hep-th/9807157;

10. V. I. Shevchenko and Yu. A. Simonov, Phys. Rev. Lett. 85, 1811 (2000) arXiv:hep-ph/0001299.

11. A. Di Giacomo, H. G. Dosch, V. I. Shevchenko and Yu. A. Simonov, Phys. Rept. 372, 319 (2002) arXiv:hep-ph/0007223.

12. Yu. A. Simonov, Few Body Syst. 25, 45 (1998) arXiv:hep-ph/9712248.

13. Yu. A. Simonov, Phys. Lett. B 226, 151 (1989).

14. A. Yu. Dubin, A. B. Kaidalov and Yu. A. Simonov, Phys. Lett. B 323, 41 (1994).

15. A. Yu. Dubin, A. B. Kaidalov and Yu. A. Simonov, Phys. Atom. Nucl. 56, 1745 (1993) [Yad. Fiz. 56, 213 (1993)] arXiv:hep-ph/9311344.

16. Yu. A. Simonov, Phys. Lett. B 515, 137 (2001) arXiv:hep-ph/0105141.

17. A. M. Badalian, B. L. Bakker and Yu. A. Simonov, Phys. Rev. D 66, $034026 \quad$ (2002) arXiv:hep-ph/0204088.

18. A. L. Kataev, N. V. Krasnikov and A. A. Pivovarov, Phys. Lett. B 123, 93 (1983);

S. G. Gorishnii, A. L. Kataev and S. A. Larin, Phys. Lett. B 135, 457 (1984). 